Supporting information

\title{
Synthesis of polyphosphoesters
}

The ring-opening polymerization of EP and IPP was performed by a method previously described. ${ }^{1}$ Briefly, a given amount of EP and IPP were placed into a thoroughly dried 50-ml round-bottomed flask equipped with a three-way stopcock. After the mixture was dried under reduced pressure for $2 \mathrm{~h}$, triisobutyl aluminum was added under an argon gas atmosphere. The reaction was continued until the magnetic stirrer was stopped. Dry THF was then added to dilute the reaction mixture. The polyphosphate $\left(\mathrm{PI}_{\mathrm{x}} \mathrm{E}_{\mathrm{y}} \mathrm{P}\right)$ was purified by reprecipitation from diethyl ether. Table 1 lists the synthetic results of the $\mathrm{PI}_{\mathrm{x}} \mathrm{E}_{\mathrm{y}} \mathrm{P}$. The weight-averaged molecular weights was determined by gel-permeation chromatography through a Polymer Laboratories MIXED-C column using a calibration curve based on linear polystyrene standards. Chloroform was the GPC solvent.

Table s-1 Synthetic results of PIEP

\begin{tabular}{|c|c|c|c|c|}
\hline \multirow{2}{*}{ Polymer } & \multicolumn{2}{|c|}{ Mole fraction (IPP/EP) } & \multirow{2}{*}{$\mathrm{Mw}\left(10^{4}\right)^{2)}$} & \multirow{2}{*}{$\mathrm{Mw} / \mathrm{Mn}^{2)}$} \\
\hline & In feed & In copolymer ${ }^{1)}$ & & \\
\hline $\mathrm{Pl}_{16} \mathrm{E}_{84} \mathrm{P}$ & $0.30 / 0.70$ & $0.16 / 0.84$ & 1.23 & 1.19 \\
\hline $\mathrm{Pl}_{24} \mathrm{E}_{76} \mathrm{P}$ & $0.40 / 0.60$ & $0.24 / 0.76$ & 1.26 & 1.20 \\
\hline $\mathrm{PI}_{51} \mathrm{E}_{49} \mathrm{P}$ & $0.70 / 0.30$ & $0.51 / 0.49$ & 1.46 & 1.14 \\
\hline PEP & $-/ 1.00$ & $-/ 1.00$ & 1.46 & 1.25 \\
\hline
\end{tabular}

1) Determined by ${ }^{1} \mathrm{H}$ NMR

2) Determined by GPC 


\section{Determination of thermoresponsive properties}

The thermoresponsive properties of polyphosphoesters were investigated with a JASCO V-650 UV-Vis spectrophotometer. $\mathrm{PI}_{\mathrm{x}} \mathrm{E}_{\mathrm{y}} \mathrm{P}$ was dissolved in milli-Q water (1 wt\%) and a transmittance curve through the solution at a wavelength of $500 \mathrm{~nm}$ was recorded with temperature variation $\left(1^{\circ} \mathrm{C} / \mathrm{min}\right)$ between $15^{\circ} \mathrm{C}$ and $50^{\circ} \mathrm{C}$.

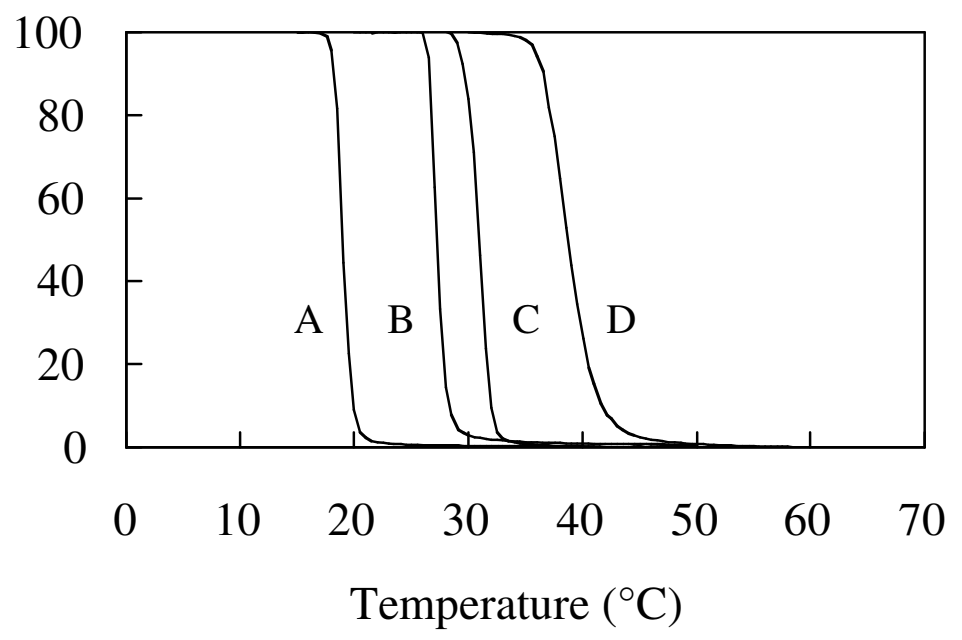

Figure s-1. Effect of temperature on light transmittance of 1 wt $\% \mathrm{PI}_{\mathrm{x}} \mathrm{E}_{\mathrm{y}} \mathrm{P}$ aqueous solution on the heating process. A: $\mathrm{PI}_{51} \mathrm{E}_{49} \mathrm{P}, \mathrm{B}: \mathrm{PI}_{24} \mathrm{E}_{76} \mathrm{P}, \mathrm{C}: \mathrm{PI}_{16} \mathrm{E}_{84} \mathrm{P}$, D:PEP.

References

1. Y. Iwasaki et al., Macromolecules 2004, 37, 7637. 